\title{
Especial
}

\section{La mediación estratégica en los conflictos psicosociales en el trabajo}

\section{The strategic mediation in the conflicts psicosociales in the work}

\section{Fernando Mansilla Izquierdo.}

Madrid-Salud. Ayuntamiento de Madrid. Madrid. España

Recibido: 21-06-12

Aceptado: 11-07-12

\section{Correspondencia}

Fernando Mansilla Izquierdo

Madrid-Salud. Ayuntamiento de Madrid

Francos Rodríguez, s/n. Dehesa de la Villa

28047 Madrid. España

Tfnos: $915132616 / 619228041$

E-mail: mansillaif@madrid.es

Resumen

La mediación es un espacio de comunicación que ha sido definida como una zona intermedia que promueve nuevas relaciones que permite la regulación de tensiones y que puede resolver de conflictos.

La mediación estratégica en salud laboral tiene que apoyar el funcionamiento psicolaboral del trabajado, porque la intervención estratégica en los conflictos interpersonales en el trabajo tiene que conducir a los trabajadores a la percepción de que ellos mismos pueden gestionar sus problemas y generar el cambio.

Med Segur Trab (Internet) 2012; 58 (228) 179-185

Palabras clave: mediación, conflicto interpersonal en el trabajo, intervención estratégica.

\begin{abstract}
The mediation is a space of communication that has been defined as an intermediate zone that promotes new relations that the tension regulation allows and that it can solve of conflicts. The strategic mediation on labor health has to rest the functioning psicolaboral of the worn out one, because the strategic intervention in the interpersonal conflicts in the work has to lead the workers to the perception of which they themselves can manage his problems and generate the change.
\end{abstract}

Med Segur Trab (Internet) 2012; 58 (228) 179-185

Key words: mediation, interpersonal conflict in the work, strategic intervention. 


\section{INTRODUCCIÓN}

La mediación es un espacio de comunicación ${ }^{21}$, y ha sido definida como una zona intermedia que promueve nuevas relaciones, como un territorio abierto para evitar problemas, como un lugar dinámico que permite la regulación de tensiones. Asimismo, se ha visto la mediación como un procedimiento de resolución de conflictos que consiste en la intervención de una tercera parte imparcial sin poder de decisión a la que aceptan las partes en conflicto, y que tiene como objetivo facilitar que se llegue a un acuerdo por medio del diálogo y de la negociación ${ }^{6}$. Así, la mediación permite resolver conflictos de manera pacífica, a través del diálogo, donde el mediador como tercero neutral y las partes en conflicto, encuentren las soluciones más beneficiosas.

La mediación debe contemplarse en la organización y en las políticas de recursos humanos de la empresa, porque es especialmente adecuada para resolver los problemas entre trabajadores o pequeños grupos de trabajadores o en procedimientos disciplinarios.

Se han diferenciado tres modelos de mediación ${ }^{22}$ : el modelo tradicional-lineal de Harvard que tiene la finalidad de conseguir acuerdos y se centra en el contenido de comunicación, en el mensaje en sí y no tiene en consideración la relación entre las partes; el modelo transformativo ${ }^{2}$ que no se centra en lograr acuerdos ni en la resolución del conflicto, sino en la transformación de las relaciones y, por último, el modelo circularnarrativo ${ }^{3}$ cuyo interés está tanto en lograr acuerdos como en la transformación de las relaciones.

La mediación como sistema de resolución de conflictos se ha desarrollado en España en el ámbito laboral y posteriormente en el ámbito familiar, comunitario y penal. Y se ha convertido en las últimas décadas en un sistema de gestión y resolución pacífica de conflictos por sus consecuencias en la mejora de la convivencia de las personas y de la sociedad $^{9,19}$. Cualquier diferencia que surge en el lugar de trabajo puede beneficiarse de la mediación si las partes están dispuestas a tratar directamente unas con otras. Es decir, viene a ser un sistema donde las personas en conflicto, ante un mediador, intentan alcanzar un acuerdo que termine total o parcialmente con su controversia.

En la mediación se pueden realizar preguntas abiertas, cerradas y circulares; también pueden replantearse situaciones que abran la posibilidad que permita modificar las posiciones de las partes.

Hay que tener presente que la mediación es un acto cooperativo no competitivo, que está más orientado al futuro que hacia el pasado, que no hay parte ganadora ni parte perdedora, que exige honestidad y franqueza, que es un proceso voluntario, que no existe el castigo y que es creativo y requiere la aportación de nuevas ideas y enfoques.

El proceso de mediación tiene que ser flexible y adaptable a las circunstancias individuales, pero cada trabajador interesado tendrá la oportunidad de dar su versión de los hechos y de aportar la solución que crea más oportuna. Además todas las personas involucradas tienen que sentirse cómodas con el proceso y en la exploración activa de las posibles soluciones ${ }^{24}$, por lo que se necesita la creación de una atmósfera de cooperación entre los trabajadores.

Para ejecutar la mediación se debe preparar el escenario, es decir el espacio físico donde se realizarán las reuniones. Durante el proceso tienen que realizarse reuniones conjuntas o reuniones del mediador con cada parte.

La mediación, pues, tiene que realizarse en un lugar neutral y en posición de igualdad; se tiene que mantener la confidencialidad y el respeto, no se delega en una tercera persona, y en la toma de decisiones se establece un turno de palabra; tiene que haber un número fijo de reuniones y tiene que controlarse la duración de cada sesión. Al final de cada reunión, los involucrados en la mediación tendrán a su disposición un resumen del mediador sobre los resultados de la investigación hecha hasta ese momento. 
Cuando el conflicto interpersonal en el trabajo se intensifica, la comunicación se hace difícil y las relaciones resultan dañadas, por lo que involucrar a un tercero independiente ayuda a negociar y a encontrar un punto de acuerdo. Y la mediación tiene lugar cuando las partes en la disputa reconocen su existencia, acuerdan la necesidad de resolverla, y se comprometen activamente en un proceso diseñado para solucionar la misma.

La mediación en los conflictos laborales es el camino hacia el futuro y debería ser una técnica incluida en todas las organizaciones, ya que fomenta el diálogo y la convivencia entre los trabajadores, a la vez que contribuye a la transformación pacífica de las dinámicas conflictivas en el lugar de trabajo, porque la mediación ofrece a los miembros de una organización las herramientas para la intervención temprana y la resolución de las controversias $^{24}$.

Con la mediación hay más posibilidades de solución cuando los conflictos son recientes y cuando las partes tienen más opciones a su disposición para la resolución ${ }^{24}$.

La resolución de los conflictos se refiere a restaurar las relaciones de trabajo saludable, a la búsqueda de una alternativa imparcial, a la superación de los obstáculos que se presentan, y a los procesos que implican los caminos que conducen a los acuerdos y desacuerdos ${ }^{7}$ a través de la negociación y del apoyo.

Y cada vez más se insiste en la mediación para resolver conflictos, para lograr acuerdos, para evitar el litigio y para restablecer las relaciones deterioradas. Incluso la mediación en el lugar de trabajo se está convirtiendo en un método de solución de controversias para resolver los conflictos interpersonales de los empleados, incluyendo el acoso y la intimidación ${ }^{4}$.

\section{MATERIAL Y MÉTODOS}

\section{a. Conflicto laboral}

La vida laboral puede ser una experiencia enriquecedora para el trabajador o, por el contrario, puede convertirse en una vivencia frustrante, aunque para una mayoría de trabajadores puede convertirse en una combinación de ambas en diferentes etapas de la historia laboral.

La interacción en el trabajo puede dar lugar a conflictos por las diferencias individuales, por la percepción de actitudes inadecuadas, por la competencia, por la rivalidad, por la necesidad de status, etc.

Los conflictos laborales entre los trabajadores y su gestión ineficaz pueden dar lugar un quebranto de la calidad de vida laboral y a la reducción de la productividad ${ }^{18}$. De ahí que los directivos dediquen más del $20 \%$ de su tiempo en gestionar estos conflictos y en buscar soluciones para prevenir o paliar sus efectos ${ }^{23}$. Una forma de gestionar los conflictos entre los trabajadores es la mediación.

Cuando se da un conflicto entre los trabajadores, el mediador tiene la función de facilitar la comunicación entre estos últimos para que, mediante el diálogo, lleguen a resolverlo. Aunque el mediador no tiene la función o responsabilidad de solucionar el conflicto, sí tiene que posicionarse en la intervención, para que las personas en conflicto dialoguen con una construcción narrativa diferente.

La mediación puede incluir desde los conflictos laborales, las situaciones de estrés laboral, el síndrome de burnout, el mobbing, las conductas de acoso sexual y el deterioro del rendimiento del trabajador que las evaluaciones de riesgos psicosociales pueden poner de manifiesto.

Por ello, el Servicio de Prevención de Riesgos Laborales debería tener la facultad de llevar a cabo la mediación como institución regulada, es decir, podría proponer al 
mediador y a los expertos en las diversas materias que se requieran, tanto para realizar encuestas como estudios, porque el encargado de realizar la mediación tendría que ser también un profesional de Vigilancia de la Salud o Especialista en Psicosociología Laboral que debería poseer cualidades como la empatía, la imparcialidad, la confidencialidad, la autoridad y la profesionalidad para el desempeño del rol de mediador y la diligencia para realizar propuestas independientes, ya que se ha demostrado la eficacia en la resolución de conflictos de mediadores laborales ${ }^{20}$.

El conflicto interpersonal en el trabajo puede tener como protagonistas a jefes y trabajadores, también se puede dar entre compañeros o puede tomar la forma de rivalidad entre los equipos y entre grupos de trabajadores. Es decir, hace referencia a situaciones en las que dos o más partes están en desacuerdo entre sí. Pero el desacuerdo dentro de la organización, generalmente, es multicausal, puede generar ineficiencia e inefectividad, aunque también, puede hacer crecer la organización ${ }^{8}$.

\section{b. Mediación estratégica}

La mediación con enfoque estratégico se basa en la teoría sistémica a la que interesa la organización del sistema y las interacciones entre individuos. También se basa en el constructivismo para el que no existe una realidad sino tantas realidades que varían según el punto de vista que se escoge para observarla. Así mismo se basa en la concepción unitaria de la persona de Milton Ericsson y que contempla las limitaciones y la resistencia al cambio no como un obstáculo sino como una fuerza orientada a la solución.

La mediación estratégica en salud laboral tiene que apoyar el funcionamiento psicolaboral del trabajador y solucionar cualquier conflicto interpersonal. Y está enfocada a modificar la representación que el trabajador tiene de su problema y de su realidad, de manera que no sólo se soluciona el problema sino que se construyen nuevas herramientas a disposición de los trabajadores para enfrentarse de un modo más eficaz a sus propias vidas.

La mediación estratégica intenta resolver en el menor tiempo posible los conflictos o problemas que pueden ser disfuncionales, porque una característica de este modelo es su capacidad de producir cambios en tiempos breves, incluso en problemas enraizados y persistentes durante años.

El principal instrumento de la mediación estratégica es el diálogo estratégico, mediante el cual se puede llegar a una percepción alternativa de la realidad y a descubrir el modo de resolver los problemas y dificultades de una persona en relación consigo misma, con los demás y con el mundo ${ }^{11,16}$. También se utilizan tareas, prescripciones, redefiniciones, reformulaciones, desafíos, paradojas, metáforas, aforismos u otras herramientas, que de forma directa o indirecta, intenta que el trabajador encuentre su propio camino hacia el cambio ${ }^{12,13,17}$.

Se tienen que inducir cambios en las modalidades con las que los trabajadores han construido realidades disfuncionales, partiendo de la premisa de que la mente humana es activa y constructiva, esto es, crea la realidad que después sufre. Y llegar a una redefinición cognitiva y a experimentar percepciones alternativas de la realidad, con el objetivo de promover la construcción de un nuevo sistema perceptivo-reactivo, que facilita las relaciones con uno mismo y con los demás. Un cambio en la percepción de la realidad del trabajador llevará a cambiar sus reacciones a nivel emocional, cognitivo y conductual ${ }^{15}$.

En ocasiones el trabajador lleva a cabo una serie de acciones que en lugar de resolver el problema pueden llevar a que la situación problemática se mantenga inalterable, creando un círculo vicioso en el que las soluciones intentadas forman parte del problema y lo alimentan. Es decir, que estas soluciones intentadas que el trabajador pone en marcha en lugar de resolver el problema lo mantienen o lo agravan ${ }^{5}$.

Si se bloquean o se cambian las soluciones intentadas disfuncionales se interrumpe el círculo vicioso que alimenta la persistencia del problema, abriendo el camino a un 
cambio real y alternativo (a nivel emocional, cognitivo y conductual), basado en nuevas percepciones de la realidad ${ }^{14}$.

La palanca de cambio es la solución intentada, porque es ésta la que ha convertido una dificultad en un problema, es decir la dinámica de la relación conflictiva entre los trabajadores.

Es crucial determinar la solución intentada para desechar aquellas soluciones que no son eficaces, para no sugerir soluciones que ya se han intentado; si se está intentando una solución exitosa, sólo es necesario apoyarla ${ }^{14}$.

Cualquier conflicto que surja en el lugar de trabajo puede beneficiarse de la mediación si las partes están dispuestas a tratar directamente unas con otras y si la empresa tiene un mediador.

La mediación estratégica es de tipo activa y prescriptiva; es decir, se prescriben o indican una serie de tareas que el trabajador tiene que realizar

y, a medida que las realiza, va haciendo nuevos descubrimientos sobre sus capacidades de cambio. Además, hay que utilizar un tipo de comunicación inductivo y sugestivo, formulando preguntas que permitan al trabajador descubrir aspectos que le habían pasado inadvertidos, lo que ayuda a crear una atmósfera sugestiva y saber que la relación va dirigida siempre al cambio. Una vez que se ha producido el cambio y con objeto de que los trabajadores se responsabilicen, la comunicación se vuelve menos sugestiva y más de confrontación, para que emerja la confianza en los recursos personales.

Además se trata de educar acerca de los mecanismos que desencadenan y mantienen el problema. Esta información permite reinterpretar los problemas. De manera que se pueda bloquear la situación y generar un cambio. Porque se han bloqueado las soluciones intentadas para solucionar los problemas, que mantenían o exacerbaban el problema ${ }^{10}$.

Si las emociones de los trabajadores que están en conflicto les hacen centrarse en el pasado y en lo negativo, con el proceso de mediación se trata de cambiar esta situación, haciendo que las partes se centren en el presente y en lo positivo mediante la disminución de la intensidad emocional, mejora en la comunicación y cambios en la manera de comprender el punto de vista del otro ${ }^{1}$.

Hay que reconocer los sentimientos que se experimentan a partir de los problemas e identificar las pautas relación disfuncionales en que se encuentran los trabajadores, y analizar formas de interrumpir o introducir algún cambio en las pautas de relación. Se busca explorar los recursos de los trabajadores para que puedan construir nuevas formas de resolver los problemas. Un pequeño cambio significativo en la vida de los trabajadores puede llevar a grandes cambios. Se trata de que se genere un contexto cognitivo, actitudinal y emocional en el cual el problema pierda su razón de ser y desaparezca como tal. De modo que se pueda construir otro mapa de relaciones interpersonales entre los trabajadores, porque se sienten comprendidos y convencidos de que ellos llevan las riendas. Esto se realiza en un proceso indirecto, sin dirigismo, a través de reencuadres o reformulaciones. También se pueden emplear diversas técnicas de comunicación como el parafraseo, las redefiniciones, etc., a fin de concretar y redefinir el contenido de lo relacional.

Los trabajadores tienen que explorar las creencias que sostienen ciertas formas problemáticas de interactuar en la relación, y trabajar a partir de ellas, haciéndolas explicitas, revisándolas y cuestionándolas; arriesgándose a tomar la iniciativa y reaccionar de manera diferente ante la situación que ha sido un problema para su relación, lo que puede abrir la posibilidad de que se obtengan resultados distintos.

Ante los conflictos interpersonales pueden generarse diferentes alternativas y la mediación estratégica puede ofrecer una oportunidad para que los involucrados exploren activamente las posibles soluciones, resuelvan problemas y lleguen a un resultado factible a través de un proceso flexible que pueda adaptarse a la situación particular. 
Cuando se reconocen y aceptan los sentimientos, cuando se posibilita que la relación con el otro se vaya construyendo sobre la base de lo que se observa, cuando se da un nuevo comportamiento, cuando se presta atención a lo que se nos comunica con las palabras y acciones, se abre la posibilidad de que ocurran cambios. Porque se trata de superar los propios patrones problemáticos mediante nuevas experiencias y formas de entender la vida laboral, cambiando la forma en que el trabajador se relaciona consigo mismo y con los demás trabajadores. Es decir, se trata del cambio de patrones y esquemas.

En definitiva, la intervención debe discurrir en dos fases. En la primera fase: "desbloquear la problemática", redefiniendo la posibilidad de cambio, estimulando cambios progresivos y si no se produce ningún cambio, utilizando estrategias para detener las soluciones intentadas fallidas y para empezar a cambiar el rígido sistema perceptivo-reactivo. Y en la segunda fase: "consolidar y reorganizar las reglas de la relación”, con cambios progresivos hasta alcanzar los objetivos y adquirir una percepción de la realidad y una reacción flexible ante ella.

Así pues, la intervención estratégica en los conflictos interpersonales en el trabajo desde este enfoque debe ser realizada por profesionales con cierta experiencia en este tipo de intervenciones, y que conozcan los procesos intrapersonales e interpersonales que generan tales situaciones, ya que son éstos quienes tienen que definir las estrategias más adecuadas para solucionar el conflicto.

\section{DISCUSIÓN Y CONCLUSIONES}

Dijo Oscar Wilde que existen tantas realidades como puedan inventarse, lo que en el mundo laboral tiene un reflejo adecuado por las realidades laborales cambiantes, y porque el arte de cambiar situaciones problemáticas aplicando estrategias forma parte de una tradición milenaria ${ }^{15}$.

Porque son los trabajadores los que solucionan sus problemas y son capaces de encontrar los mecanismos para resolver las situaciones que antes les perjudicaban ${ }^{14}$, ya que la intervención estratégica en los conflictos interpersonales en el trabajo tiene que conducir a los trabajadores a la percepción de que ellos mismos pueden gestionar sus problemas y generar el cambio.

Sin duda, es el enfoque estratégico tiene un aspecto pragmático y trabaja la comunicación entre los trabajadores que quieren gestionar las diferencias, buscando por ellas mismas soluciones a sus conflictos por la vía del diálogo ${ }^{11,14}$, por lo que la intervención estratégica requiere profesionales que manejen este tipo de intervenciones.

\section{REFERENCIAS BIBLIOGRÁFICAS}

1. Bernal T. La mediación. Una solución a los conflictos de ruptura de pareja. Madrid: Colex, 2002.

2. Bush RAB, Folger JP. La promesa de la mediación. Cómo afrontar el conflicto a través del fortalecimiento y el reconocimiento de los otros. Barcelona: Gránica; 1996.

3. Cobb S (1993). Empowerment and Mediation: A Narrative Perspectiva. Negotiation Journal 1993; 9 (3): $245-255$.

4. Doherty N, Guyler M. The Essential Guide to Workplace Mediation and Conflict Resolution: Rebuilding Working Relationships. London: Kogan Page, Ltd; 2008.

5. Hirsch H. Qué y cómo: Prácticas en psicoterapia estratégica. Buenos Aires: Dunken; 2007.

6. Jares XR. Educación y conflicto. Guía de educación para la convivencia. Madrid: Popular; 2001.

7. Kindler HS. Cómo gestionar los conflictos. Madrid: Centro de Estudios Ramón Areces; 2001.

8. Mansilla F. Manual de riesgos psicosociales en el trabajo: Teoría y práctica. Disponible http://www. psicologia-online.com/ebooks/riesgos/index.shtml.; 2012

9. Martinez-Pecino R, Munduate L, Medina FJ (2008). La gestión de conflictos organizacionales por medios extrajudiciales. Papeles del Psicólogo 2008; 29: 41-48. 
10. Nardone G. Psicosoluciones. Cómo resolver rápidamente problemas humanos. Barcelona: Herder; 2002.

11. Nardone G. Corrígeme si me equivoco. Estrategias de diálogo en la pareja. Barcelona: Herder; 2006.

12. Nardone G. La mirada del corazón. Aforismos terapéuticos. Barcelona: Paidós; 2008.

13. Nardone G. La dieta de la paradoja. Cómo superar las barreras psicológicas que te impiden adelgazar y estar en forma. Barcelona: Paidós; 2009.

14. Nardone G. Problem Solving Estratégico. El arte de encontrar soluciones a problemas irresolubles. Barcelona: Herder; 2010.

15. Nardone G, Portelli C. Conocer a través del cambio. Barcelona: Herder; 2006.

16. Nardone G, Salvini A. El diálogo estratégico. Comunicar persuadiendo: técnicas para conseguir el cambio. Barcelona: Herder; 2011.

17. Nardone G, Watzlawick P. Terapia Breve: filosofía y arte. Barcelona: Herder: 2003.

18. Roberts T. Coaching managers through their conflicts. Management Services 2005; 49: 16-18.

19. Serrano G. Eficacia y mediación familiar. Boletín de Psicología 2008; 92: 51-63.

20. Serrano G, Lopes C, Rodríguez D, Mirón L. Características de los mediadores y éxito de la mediación. Anuario de Psicología Jurídica 2006; 16: 75-88.

21. Six J F. Dinámica de la mediación. Barcelona: Paidós; 1996.

22. Suares M. La Mediación. Conducción de disputas, comunicación y técnicas. Barcelona: Paidós; 1999.

23. Ursiny T, Bolz D. The top performer's guide to conflict. Naperville: Sourcebooks, Inc; 2007.

24. Weinstein RJ. Mediation in the workplace. A Guide for Training, and Administration. Westport: Greewood Publising Group, Inc; 2001.

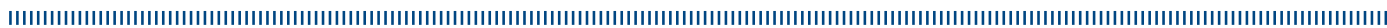

